\title{
PI3K/AKT/PTEN pathway as a target for Crohn's disease therapy (Review)
}

\author{
NANA TOKUHIRA*, YASUKO KITAGISHI*, MIHO SUZUKI, AKARI MINAMI, ATSUKO NAKANISHI, \\ YUNA ONO, KEIKO KOBAYASHI, SATORU MATSUDA * and YASUNORI OGURA
}

Department of Food Science and Nutrition, Nara Women's University, Nara 630-8506, Japan

Received June 13, 2014; Accepted October 16, 2014

DOI: $10.3892 / \mathrm{ijmm} .2014 .1981$

\begin{abstract}
The pathogenesis of inflammatory bowel disease (IBD), including Crohn's disease, is a subject of increasing interest. Loss-of-function mutations in nucleotide-binding oligomerization domain-containing protein 2 (NOD2) are strong genetic factors linked to Crohn's disease, which eventually leads to an excessive mucosal inflammatory response directed against components of normal gut microbiota. Reactive oxygen species (ROS) play an important role in inflammation processes, as well as in transduction of signals from receptors for several cytokines, such as tumor necrosis factor $\alpha(\mathrm{TNF} \alpha)$. ROS activate nuclear factor- $\kappa \mathrm{B}(\mathrm{NF}-\kappa \mathrm{B})$ via I $\mathrm{B}$ kinase (IKK) through the PI3K/AKT/PTEN pathway. Therefore, this pathway is recognized to play a key role in Crohn's disease. Loss of function has been demonstrated to occur as an early event in a wide variety of diseases. Given this
\end{abstract}

Correspondence to: Professor Satoru Matsuda, Department of Food Science and Nutrition, Nara Women's University, Kita-Uoya Nishimachi, Nara 630-8506, Japan

E-mail: smatsuda@cc.nara-wu.ac.jp

*Contributed equally

Abbreviations: CARD, caspase recruitment domain; FKBP12, FK506-binding protein 12; GSK, glycogen synthase kinase; IBD, inflammatory bowel disease; IKK, IKB kinase; LRR, leucine-rich repeat; MDP, muramyl dipeptide; mTOR, mammalian target of rapamycin; MyD88, myeloid differentiation factor 88; NF- $\kappa \mathrm{B}$, nuclear factor- $\kappa \mathrm{B}$; NLR, nucleotide-binding oligomerization domain and LRR-containing protein; NOD2, nucleotide-binding oligomerization domain-containing protein 2; PDK1, phosphoinositide-dependent kinase 1; PDZ, PSD-95, DLG1 and ZO-1; PEST, proline, glutamic acid, serine and threonine; $\mathrm{PH}$, pleckstrin homology; PI3K, phosphatidylinositol 3-kinase; PIP3, phosphatidylinositol 3,4,5triphosphate; PTEN, phosphatase and tensin homolog; RTKs, receptor tyrosine kinases; SMA, 7- $O$-succinyl macrolactin A; TLRs, Toll-like receptors; TNF, tumor necrosis factor; TSC1, tuberous sclerosis complex 1; TSC2, tuberous sclerosis complex 2

Key words: Crohn's disease, nucleotide-binding oligomerization domain-containing protein 2, phosphatidylinositol 3-kinase, AKT, mammalian target of rapamycin, phosphatase and tensin homolog, inflammation prevalent involvement in a number of diseases, the molecular development that modulates this pathway has been the subject of several studies. In addition, it has been the focus of extensive research and drug discovery activities. A better understanding of the molecular assemblies may reveal novel targets for the therapeutic development against Crohn's disease.

\section{Contents}

1. Introduction

2. Key molecular signaling in Crohn's disease

3. PI3K/AKT/mTOR signaling involved in NOD2-mediated pathway

4. Possible modulators of the PI3K/AKT pathway against Crohn's disease

5. Effect of diet on PI3K/AKT/PTEN pathway and possible restorative effects in patients with Crohn's disease

6. Perspectives

\section{Introduction}

In the human gut, a continuous homeostasis is maintained by the strict regulation of microbial load and the immune responses against it $(1,2)$. Under normal physiological conditions, gut microbiota colonize and contribute to the proper development of the mucosal immune system $(1,2)$. When the epithelial barrier is disrupted, they induce an uncontrollable inflammatory condition. The breakdown of this balance by the dysregulation of immune responses may increase susceptibility to chronic inflammatory disorders, such as Crohn's disease $(3,4)$. Uncontrolled mucosal inflammatory responses against gut microbiota due to the disruption of the epithelial barrier hence play important roles in the pathogenesis of Crohn's disease $(3,4)$. Crohn's disease is a chronic inflammatory disorder associated with mucosal inflammation of the bowel wall, which is characterized by repetitive active cycles of the disease state (5). It is histologically characterized by the massive transmural infiltration of lymphoctes and macrophages with granulomas (6). Crohn's disease can affect the entire gastrointestinal tract although the most common presentation is the ileum-colon junction. Pro-inflammatory cytokines, such as tumor necrosis factor $\alpha(\mathrm{TNF} \alpha)$, are pivotal for the 
development of inflammatory bowel disease (IBD). Therefore, the downregulation of cytokines and cytokine-induced inflammatory responses constitute molecular targets for the development of therapeutic strategies in IBD (7). Numerous agents are currently available for the treatment of Crohn's disease. When treating patients with Crohn's disease, therapy is usually aimed at the effective induction and maintenance of remission, as well as in reducing therapy-related issues and improving quality of life. Anti-TNF $\alpha$ therapy is an effective therapy for Crohn's disease, and a large proportion of patients show a favorable response to its therapeutic antibodies (8). Despite the therapeutic efficacy of anti-TNF $\alpha$ agents, however, treatment failure is frequently observed. On the other hand, the macrolide, 7- $O$-succinyl macrolactin A (SMA), markedly inhibits the TNF $\alpha$-induced adhesion of monocytes to epithelial cells similar to rapamycin, an immunosuppressant macrolide and a mammalian target of rapamycin (mTOR) inhibitor (9). Importantly, SMA is more effective in the inhibition of inflammation than 5-aminosalicylic acid, the most ordinarily prescribed agent for the treatment of IBD (9). SMA also causes the suppression of TNF $\alpha$-induced phosphorylation of phosphatidylinositol 3-kinase (PI3K), AKT and mTOR, similar to the effect of rapamycin (9). Accordingly, managing the PI3K/AKT/mTOR pathway may be a good therapeutic intervention for the treatment of Crohn's disease.

\section{Key molecular signaling in Crohn's disease}

One innovation in Crohn's disease research is the identification of nucleotide-binding oligomerization domain-containing protein 2 (NOD2) as its susceptible gene $(10,11)$. NOD2 is a member of the nucleotide-binding oligomerization domain and leucine-rich repeat (LRR)-containing protein (NLR) family of cellular sensors of pathogens, which is also a member of cytosolic factors related to the regulator of apoptosis, apoptotic protease activating factor 1 (Apaf-1), a member of a class of disease resistance proteins (12). Similar to several members of the NLR family, NOD2 contains a LRR domain on its C-terminal side and two tandem caspase recruitment domains (CARDs) on its $\mathrm{N}$-terminal side. The LRR domain has a molecular structure similar to a domain found in Toll-like receptors (TLRs) (13). NOD2 appears to regulate the host response to pathogens that may be defective in certain inflammatory diseases. Genetic variation in NOD2 is associated with susceptibility to Crohn's disease (14). NOD2 activates the downstream signaling pathways, including the nuclear factor- $\kappa \mathrm{B}(\mathrm{NF}-\kappa \mathrm{B})$ pathway, which confers responsiveness to lipopolysaccharides and interacts with a mediator of $\mathrm{NF}-\kappa \mathrm{B}$ activation (15). NF- $\kappa \mathrm{B}$ is found in the cytoplasm in its inactive form with the inhibitor of $N F-\kappa B$ subunit, which is in turn regulated by $\mathrm{I} \kappa \mathrm{B}$ kinase (IKK). The phosphorylation of the Ser32 and Ser36 residues of I $\kappa$ Ba by IKK triggers a signal for the ubiquitination and degradation of I $\kappa \mathrm{Ba}$ and then the activation of NF- $\kappa \mathrm{B}(16)$. The activation of $\mathrm{NF}-\kappa \mathrm{B}$ by IKK also occurs in association with reactive oxygen species (ROS) produced by NADPH oxidase in response to the activation of receptors, such as interleukin (IL)-1 or TNF (17). Cellular ROS metabolism is firmly regulated by a variety of proteins involved in the redox mechanism with PI3K/AKT signaling (18). AKT activates IKK, which in turn stimulates p38 MAPK in the transduction of signals originating from IL-1 receptor (19). IKK may be present in the form of a complex with mTOR. The kinase may also activate IKK.

The PI3K/AKT pathway negatively regulates the NOD2-mediated NF- $\kappa$ B pathway, which may be involved in the resolution of the inflammatory responses induced by NOD2 activation (14). Accumulating evidence has revealed that the PI3K/AKT pathway acts as a pivotal determinant of cell fate regarding senescence and apoptosis, which is mediated by intracellular ROS generation (18). Muramyl dipeptide (MDP) is the minimal bioactive peptidoglycan motif common to all bacteria, which has been shown to be recognized by NOD2 and induces AKT phosphorylation (14). NOD2 is expressed in immune tissue and bacterial peptidoglycan motifs; MDP activates NOD2, inducing a reduction in AKT Ser473 phosphorylation (20). ROS also activate PI3K/AKT and inactivate phosphatase and tensin homolog (PTEN). In addition, AKT is activated as a result of IL- $1 \beta, \mathrm{TNF} \alpha$ or lipopolysaccharide receptor stimulation. The pharmacological inhibitor of PI3K and dominant-negative forms of the regulatory subunit of PI3K enhance $N F-\kappa B$ activation, while constitutive active forms of the catalytic subunit of PI3K inhibit the NF- $\kappa \mathrm{B}$ activation and their target genes (14). AKT is inhibited by protein phosphatase-2A (PP2A), which in turn may be inactivated by ROS $(19,21)$. On the other hand, ROS induction is accompanied by the activation of PI3K. AKT substrates include IKK $\alpha$, NOS, tuberous sclerosis complex (TSC)1 and 2, caspase-9, mouse double minute 2 homolog (MDM2) and glycogen synthase kinase (GSK) $3 \beta(21,22)$. Accordingly, the pathway has central signaling elements in a diverse array of cellular functions, including proliferation, migration and inflammation responses. It is therefore reasonable that the dysregulation of the PI3K/AKT pathway has been implicated in the induction and/or progression of a variety of disease states.

\section{PI3K/AKT/mTOR signaling involved in NOD2-mediated pathway}

Functional PI3K heterodimers consist of a regulatory subunit, such as p85 and a catalytic subunit, such as p110. Each PI3K forms a family which can be divided into three classes based on its structure, distribution and mechanism of activation (23). Class I PI3Ks are further divided into class IA and IB based on their different adaptors, which are activated by receptor tyrosine kinases (RTKs) and by the G-protein-coupled receptors, respectively (24). One of the substrates for class I PI3Ks is phosphatidylinositol 4,5-bisphosphate (PIP2), resulting in the production of phosphatidylinositol 3,4,5-trisphosphate (PIP3). AKT is a downstream target of PI3Ks, which belongs to the AGC family of protein kinases (25). Human AKT has three homologous members known as AKT1, AKT2 and AKT3 (26), which contain three functionally different sites, i.e., a pleckstrin homology (PH) domain, a central catalytic domain and a C-terminal hydrophobic motif (27). The binding of PI3K products to the $\mathrm{PH}$ domain results in AKT translocation to the plasma membrane where it is activated via phosphorylation by certain upstream protein kinases, such as the phosphoinositide-dependent kinase 1 (PDK1). PIP3 binds to PDK1 via the PH domains. PDK1 then phosphorylates at Thr308 of AKT1 in its kinase domain. For the full activation of AKT, further phosphorylation of Ser473 at AKT1 by 

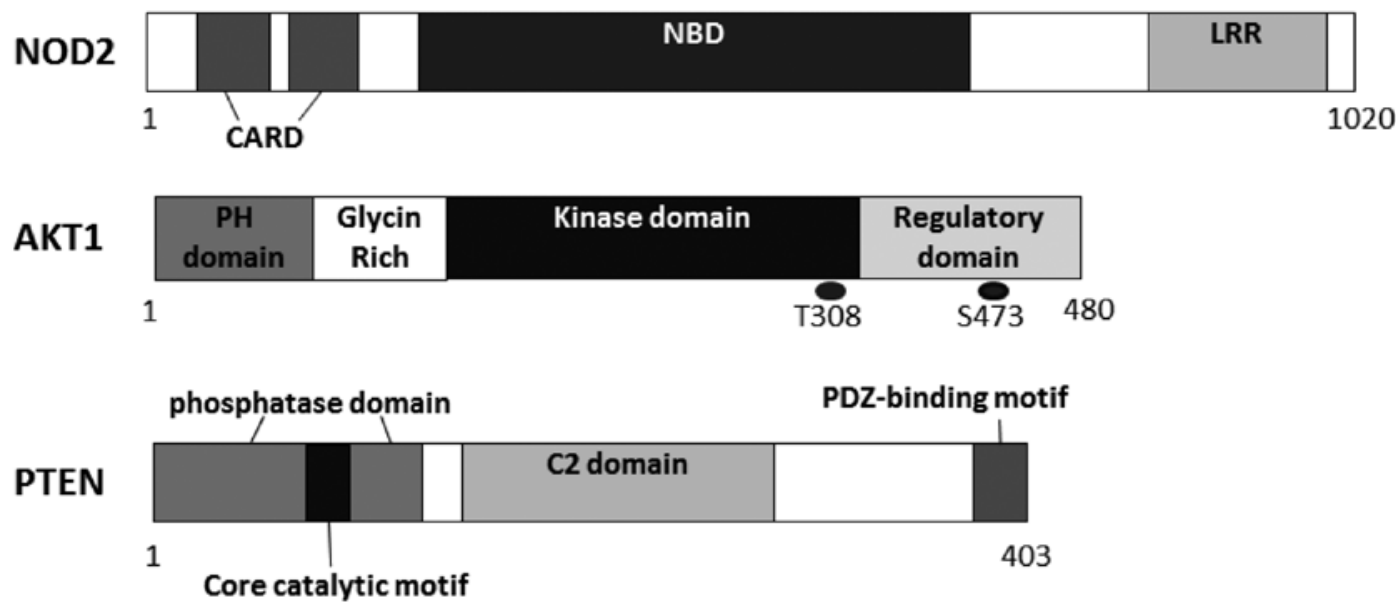

Figure 1. Schematic protein structures of human nucleotide-binding oligomerization domain-containing protein 2 (NOD2), AKT1 and phosphatase and tensin homolog (PTEN). Note that the sizes of the proteins are modified for clarity. CARD, caspase recruitment domain; NBD, nucleotide-binding domain; LRR, leucine-rich repeat; $\mathrm{PH}$ domain, pleckstrin homology domain; C2 domain, a protein structural domain involved in targeting proteins to cell membranes; PDZ, a common structural domain in signaling proteins ( $\underline{P} S D-95, \underline{D} L G 1, \underline{Z} O-1$, etc.).

PDK2 is required (28). AKT then moves to the cytoplasm and nucleus, where it phosphorylates several downstream targets to regulate several cellular functions. For example, AKT inhibits GTPase-activating protein (GAP) activity by phosphorylating TSC2, which leads to activation of the mTOR complex (29). mTOR mediates the phosphorylation of the ribosomal protein S6 kinase, leading to the release of the translation initiation factor, eukaryotic translation initiation factor 4E (eIF4E) (30). GSK3 $\beta$ is also a downstream target of AKT and is a serine/threonine kinase itself. GSK3 $\beta$ was originally identified to play a key role in the regulation of glycogen synthesis in response to insulin receptor stimulation (31), which has been shown to be involved in cellular proliferation, apoptosis and circadian entrainment, in addition to the regulation of glycogenesis (32).

The schematic structure of the predicted PTEN protein is shown in Fig. 1. The activity of PI3Ks can be inhibited by PTEN, which also has protein and lipid phosphatase activity (33). Therefore, PTEN negatively regulates AKT signaling (34). PTEN protein consists of N-terminal phosphatase, and C-terminal C2 and PSD-95, DLG1 and ZO-1 (PDZ) binding domains. The C-terminus of PTEN has two proline, glutamic acid, serine and threonine (PEST) sequences involved in protein degradation (35). PTEN can be regulated by post-translational phosphorylation, oxidation, acetylation, and so forth (36). The tissue-specific deletion of PTEN can result in autoimmunity, glucose dysregulation and/or neurological deficits. As the PI3K/AKT/mTOR/PTEN signaling axis plays a central role in metabolism and inflammation under physiological conditions (37) (Fig. 2), the effective targeting of this signaling network with pharmacological modulators may result in the effective treatment of patients with inflammation. In addition, the PI3K/AKT/mTOR/PTEN signaling pathway has been demonstrated to induce the expression of the multidrug resistance-associated protein, suggesting that high PI3K activity induces drug resistance $(38,39)$. The PI3K/AKT/mTOR signaling pathway is activated in Crohn's disease by PTEN downregulation, which may be involved in the pathogenesis this disease.

\section{Possible modulators of the PI3K/AKT pathway against Crohn's disease}

Pharmacologic modulators directed against components of intracellular signaling pathways have been developed to improve therapeutic performance. As the regulation of the components in the PI3K/AKT/PTEN pathway is thought to correlate with disease prognosis and drug resistance, it is considered to be a promising target for therapy. A number of pharmacological inhibitors of this pathway have already been developed to improve therapy (40). Usually, the PI3K/AKT/GSK3 $\beta$ activation is maintained by extracellular signals. A metabolite of guanosine released from activated $\mathrm{T}$ lymphocytes and macrophages is increased in patients with Crohn's disease (41). It has been shown that guanosine increases AKT and GSK3 $\beta$ phosphorylation (42); this suggests that it plays an important role in Crohn's disease. The cell-protective effect of guanosine is abolished by blocking the AKT pathway with LY294002 (43). Both LY294002 and wortmannin are the best characterized PI3K inhibitors which prevent ATP from binding to the active portion (44). They are low molecular weight compounds and are also cell-permeable. In addition, they enhance the phosphorylation of NF-кB p65 on Ser529 and Ser536 residues; this results in enhanced p65 transactivational activity (14). Furthermore, the inhibition of PI3K by these pharmacological inhibitors prevents the inactivation of GSK3 $\beta$ (45), suggesting that the negative regulation of $\mathrm{PI} 3 \mathrm{~K} / \mathrm{AKT}$ on $\mathrm{NF}-\kappa \mathrm{B}$ activation is mediated through the inactivation of GSK3 $\beta$. LY294002 blocks not only PI3K activity, but also mTOR to the same extent as PI3K. Some mTOR inhibitors suppress hypoxia-inducible factor $1 \alpha$ (HIF-1 $\alpha$ ) and vascular endothelial growth factor (VEGF), which initiate an inhibitory effect on the progression of inflammation (46). PI3K inhibition with LY294002 is reversible, while wortmannin irreversibly inhibits PI3K $(44,47)$. N-cadherin overexpression in bowel stricture formation in Crohn's disease may be silenced by LY294002 (48). In addition, LY294002 has been shown to reduce the production of chemokine-induced ROS in phagocytes (49). It has been reported that serum 


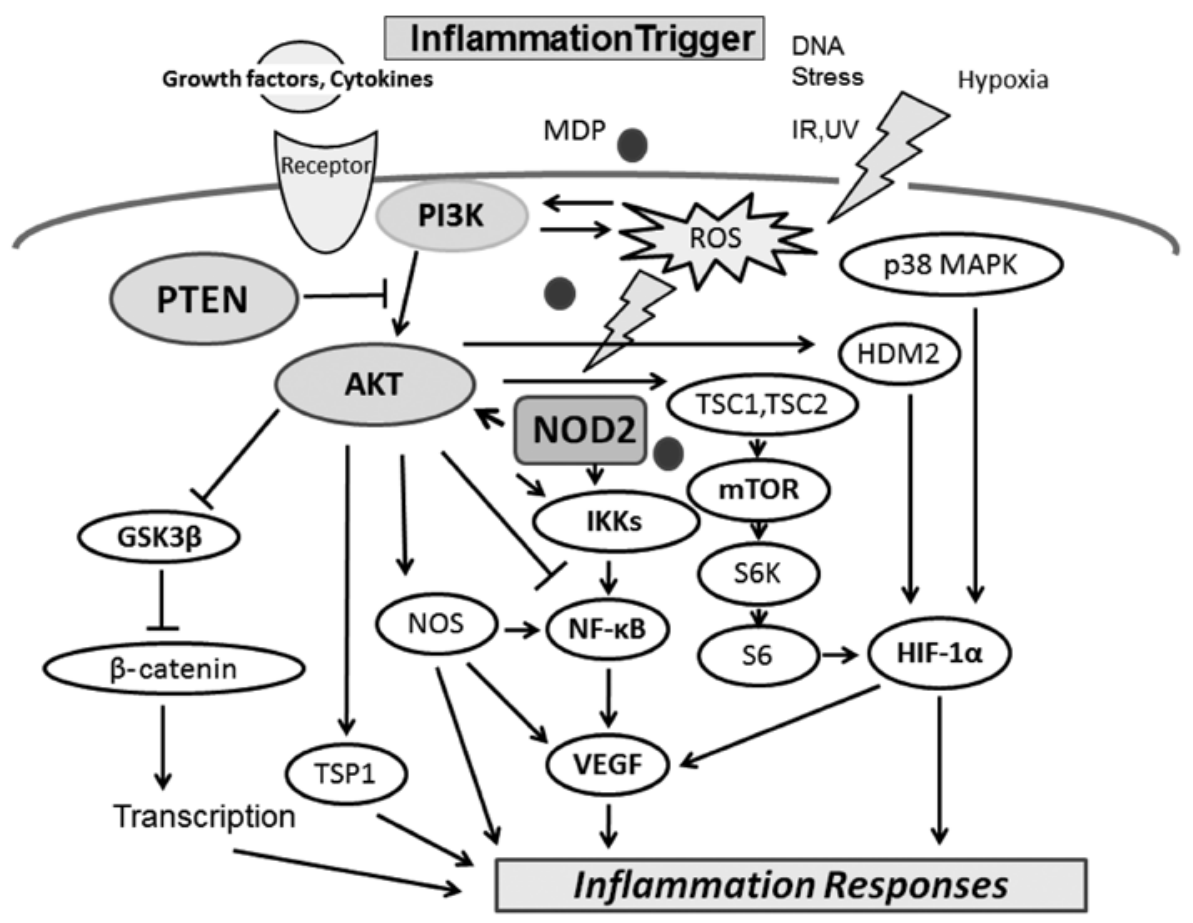

Figure 2. Schematic representation and overview of the phosphatidylinositol 3-kinase (PI3K)/AKT/phosphatase and tensin homolog (PTEN) pathway. Nucleotide-binding oligomerization domain-containing protein 2 (NOD2) senses muramyl dipeptide (MDP) leading to PI3K/AKT and nuclear factor- $\mathrm{\kappa B}$ (NF- $\mathrm{\kappa B}$ ) activation. In addition, uncontrolled generation of reactive oxygen species (ROS) contributes to the development of inflammation. Examples of molecules known to act on the PTEN/PI3K/AKT regulatory pathways are also shown. Arrowheads indicate stimulation whereas hammerheads represent inhibition. Note that some critical pathways have been omitted for clarity. ROS, reactive oxygen species; MAPK, mitogen-activated protein kinase; HDM2, human homolog of mouse double

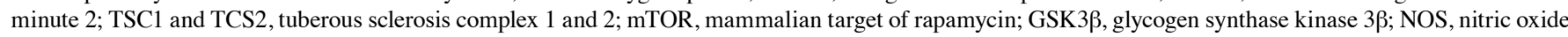
synthase; S6K, S6-kinase; HIF-1 $\alpha$, hypoxia-inducible factor 1 $\alpha$; TSP1, thrombospondin 1; VEGF, vascular endothelial growth factor.

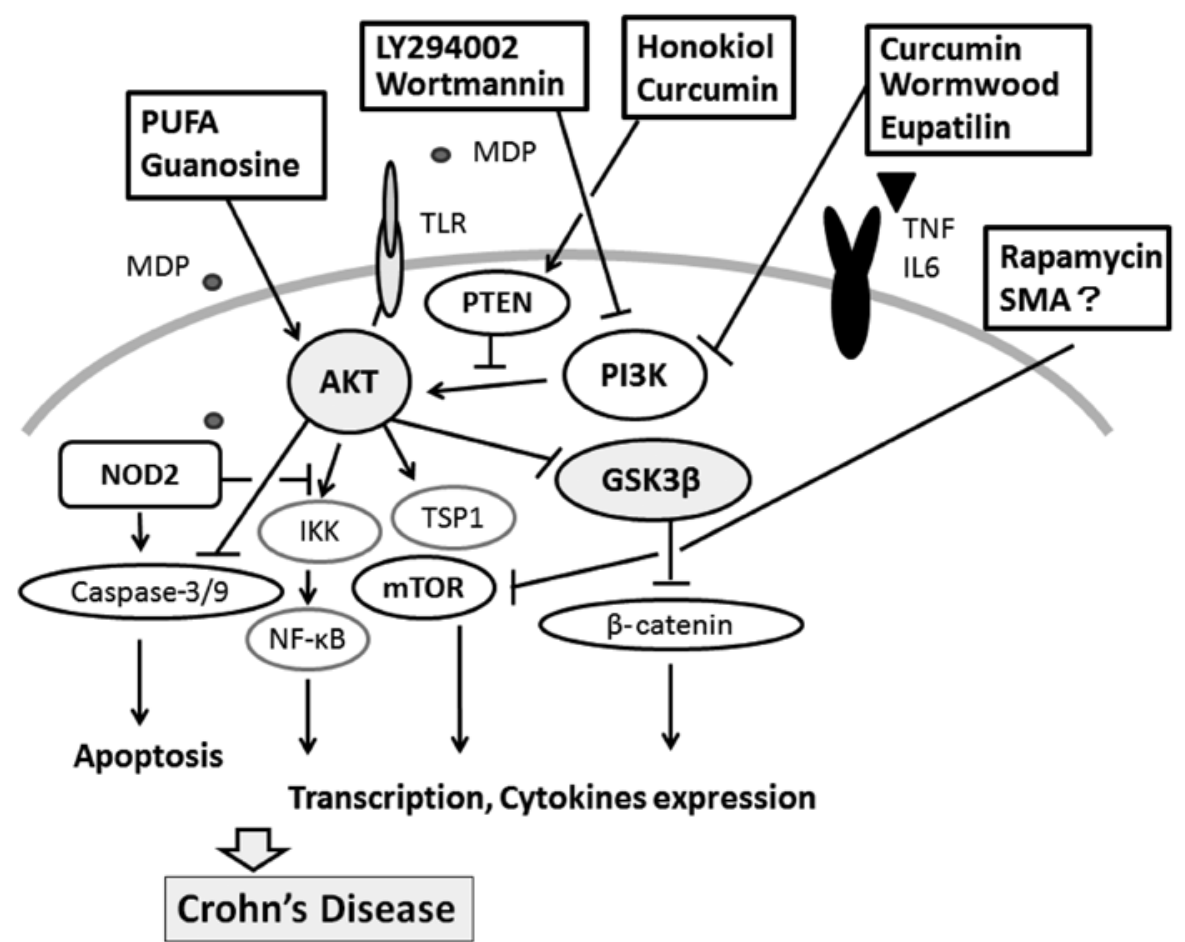

Figure 3. Several modulators linked to the phosphatidylinositol 3-kinase (PI3K)/AKT/phosphatase and tensin homolog (PTEN) signaling pathway are shown. Arrowheads indicate stimulation, whereas hammerheads represent inhibition, suggesting the possible use of PI3K/AKT/mTOR/PTEN modulators in the therapy of Crohn's disease. Potential molecular targets are based on the predominant PI3K/AKT pathway. Note that some critical events have been omitted for clarity.

withdrawal kills human U937 monocyte cells by elevating cellular ROS levels, which occurs through PI3K activation (50).
mTOR inhibitors are the most developed class of compounds including rapamycin and its derivatives, which bind to 
FK506-binding protein 12 (FKBP12) (51,52). Subsequently, the rapamycin/FKBP12 complex binds mTORC1 and prevents downstream signaling (53). ATP-competitive mTOR inhibitors suppress the activity of both mTORC1 and mTORC2 (54). Autophagy, which is a cellular process implicated in the clearance of intracellular bacteria, has been highlighted as a key feature in the pathogenesis of Crohn's disease. Rapamycin is a drug used to upregulate autophagy (55). The use of rapamycin in the treatment refractory Crohn's disease has been reported (55), offering a promising new therapeutic strategy for the treatment of IBD (56). The suppression of PTEN may increase PIP3, thus leading to the activation of PI3K/AKT signaling. However, in general, the administration of PI3K/AKT/mTOR inhibitors can give rise to potentially life-threatening adverse effects, such as pneumonitis (57).

\section{Effect of diet on PI3K/AKT/PTEN pathway and possible restorative effects in patients with Crohn's disease}

A variety of signals including growth factors and nutrients leads to PI3K/AKT pathway activation and inhibition (Fig. 3). In addition, several gene transcriptions of the components in the pathway are regulated by dietary polyunsaturated fatty acids (PUFAs) (58). Potential therapeutic strategies exploit the observation that defects in critical processes required for maintaining cellular homeostasis produce a metabolic situation characterized by Crohn's disease. It would be of significance to define appropriate strategies to achieve benefits from dietary supplements to control the activities of PI3K/AKT pathway molecules, including the expression of pro-inflammatory cytokines. Dietary supplementation of fish oil attenuates lipopolysaccharide-induced bowel inflammation (59). Fish oil increases AKT1 mRNA expressiin and decreases Forkhead Box O (FOXO)1 and FOXO4 mRNA expression (60). Fish oil also increases the phosphorylation of AKT and FOXO1. In addition, n-3-PUFAs in fish oil exert an inhibitory effect on pro-inflammatory cytokines thus affecting many inflammatory diseases $(61,62)$. In fact, linoleic acid has demonstrated efficacy as an immune modulator and anti-inflammatory compound that moderates Crohn's disease (63). Fish oil reduces the plasma levels of $\mathrm{TNF} \alpha$ and prostaglandin E2 concentrations (64). Moreover, fish oil downregulates the mRNA expression of TLR4 and its downstream signaling molecule, myeloid differentiation factor 88 (MyD88), TNFa receptor-associated factor 6 (TRAF6), NF- $\kappa \mathrm{B}$ p65 and NOD2 $(65,66)$. By suppressing pro-inflammatory cytokine production via the regulation of NOD2 signaling, fish oil may therefore improve the symptoms of Crohn's disease, possibly through the maintenance of PI3K/AKT signaling.

Several herbs may also be promising. Curcumin is an active ingredient derived from the root of the Curcuma longa plant, which has been used as a traditional Chinese herb for the treatment of various inflammatory diseases (67). Treatment with curcumin has been shown to significantly attenuate myocarditis and improve heart histopathology (68). Of note, curcumin administration reduces the expression of pro-inflammatory cytokines, such as TNF $\alpha$, IL-1 $\beta$ and IL6. Curcumin treatment also inhibits the activation of NF- $\mathrm{\kappa B}$ in a PI3K/AKT pathway-dependent manner, indicating that curcumin exerts a protective effect against inflammatory response by inhibiting the PI3K/AKT/NF-אB pathway (68). Hence, curcumin may have a therapeutic use in the prevention and treatment of Crohn's disease (69). Wormwood (Artemisia absinthium) also accelerates healing in patients with Crohn's disease (70) and has a positive effect on their mood and quality of life (71). Eupatilin, a flavonoid from wormwood, inhibits PI3K activity, causing a direct effect on the phosphorylation of downstream AKT and p70S6K (72). Licorice is a common Chinese medicinal herb with anti-tumor activity, which induces autophagy through the inhibition of the PI3K/AKT/mTOR pathway (73). Honokiol is has been demonstrated to attenuate PI3K/AKT/mTOR signaling through the upregulation of PTEN expression $(74,75)$. Curcumin also restores PTEN expression (76). By contrast, a component of the herb rosemary inhibits the expression of PTEN in the K562 myeloid cell line (77).

\section{Perspectives}

An important mediator implicated in the regulation of several diseases is PI3K/AKT/PTEN signaling. Efforts to exploit pharmacological modulators of the cascade that show efficacy and safety are in progress. It is unlikely that the regulation of a single signaling pathway will be a cure for Crohn's disease. However, the combination of regulators and conventional chemotherapeutic drugs may prove to be an effective therapeutic option for patients with this disease. Disorders are characterized by multiple signaling abnormalities and deregulated pathways may be redundant. It is difficult to find the correct combinations of accurate targets. The precise involvement of $\mathrm{PI} 3 \mathrm{~K} / \mathrm{AKT} / \mathrm{GSK} 3 \beta / \mathrm{mTOR} / \mathrm{PTEN}$ in disease signaling has not yet been fully elucidated. PTEN appears to act as a regulator of basal PIP3 levels that maintains the levels below a threshold for signaling activation, suggesting that the levels of PIP3 may have an appropriate level zone. That is the reason why both activators and inhibitors of the PI3K/AKT pathway may contribute to the treatment of Crohn's disease. Although further research is required to examine the safety and efficacy of regulators, the indicated compounds appear to possess promising therapeutic activities. An understanding of the intracellular mechanisms may provide innovative insight into the development of therapeutic approaches. To further optimize therapeutic regimens, research should also focus on the combination of regulators of PI3K/AKT signaling and regulators directed against other signal transduction molecules. Further studies are warranted to assess the safety and efficacy of these regulators using largescale cohorts of patients with IBD.

\section{Acknowledgements}

This study was supported by Grants-in-Aid from the Ministry of Education, Culture, Sports, Science and Technology in Japan.

\section{References}

1. Cénit MC, Matzaraki V, Tigchelaar EF and Zhernakova A: Rapidly expanding knowledge on the role of the gut microbiome in health and disease. Biochim Biophys Acta. pii: S0925-4439(14)00151-00153, 2014.

2. Taschuk R and Griebel PJ: Commensal microbiome effects on mucosal immune system development in the ruminant gastrointestinal tract. Anim Health Res Rev 13: 129-141, 2012. 
3. Nepal S, Navaneethan U, Bennett AE and Shen B: De novo inflammatory bowel disease and its mimics after organ transplantation. Inflamm Bowel Dis 19: 1518-1527, 2013.

4. Cromer WE, Mathis JM, Granger DN, Chaitanya GV and Alexander JS: Role of the endothelium in inflammatory bowel diseases. World J Gastroenterol 17: 578-593, 2011.

5. van Lent AU and D'Haens GR: Management of postoperative recurrence of Crohn's disease. Dig Dis 31: 222-228, 2013.

6. Irié T, Maeda Y, Aida T, Sumitani K, Nagumo M and Tachikawa T: Multiple granulomatous inflammation in the minor salivary glands: a proposed new entity, allergic granulomatous sialadenitis. Pathol Int 54: 850-853, 2004.

7. Blandizzi C, Gionchetti P, Armuzzi A, Caporali R, Chimenti S, Cimaz R, Cimino L, Lapadula G, Lionetti P, Marchesoni A, Marcellusi A, Mennini FS, Salvarani C and Girolomoni G: The role of tumour necrosis factor in the pathogenesis of immune-mediated diseases. Int J Immunopathol Pharmacol 27 (Supple 1): S1-S10, 2014

8. de Boer NKh, Löwenberg M and Hoentjen F: Management of Crohn's disease in poor responders to adalimumab. Clin Exp Gastroenterol 7: 83-92, 2014.

9. Park S, Regmi SC, Park SY, Lee EK, Chang JH, Ku SK, Kim DH and Kim JA: Protective effect of 7-O-succinyl macrolactin A against intestinal inflammation is mediated through $\mathrm{PI} 3-\mathrm{kinase} / \mathrm{Akt} / \mathrm{mTOR}$ and NF- $\mathrm{KB}$ signaling pathways. Eur J Pharmacol 735: 184-192, 2014.

10. Hugot JP, Chamaillard M, Zouali H, Lesage S, Cézard JP, Belaiche J, Almer S, Tysk C, O'Morain CA, Gassull M, Binder V, Finkel Y, Cortot A, Modigliani R, Laurent-Puig P Gower-Rousseau C, Macry J, Colombel JF, Sahbatou M and Thomas G: Association of NOD2 leucine-rich repeat variants with susceptibility to Crohn's disease. Nature 411: 599-603, 2001.

11. Ogura Y, Bonen DK, Inohara N, Nicolae DL, Chen FF, Ramos R, Britton H, Moran T, Karaliuskas R, Duerr RH, Achkar JP, Brant SR, Bayless TM, Kirschner BS, Hanauer $\mathrm{SB}$, Nuñez G and Cho JH: A frameshift mutation in NOD2 associated with susceptibility to Crohn's disease. Nature 411: 603-606, 2001.

12. Eckmann L and Karin M: NOD2 and Crohn's disease: loss or gain of function? Immunity 22: 661-667, 2005.

13. Tsai WH, Huang DY, Yu YH, Chen CY and Lin WW: Dual roles of NOD2 in TLR4-mediated signal transduction and -induced inflammatory gene expression in macrophages. Cell Microbiol 13: 717-730, 2011

14. Zhao L, Lee JY and Hwang DH: The phosphatidylinositol 3-kinase/Akt pathway negatively regulates Nod2-mediated NF-kappaB pathway. Biochem Pharmacol 75 : $1515-1525,2008$

15. Hasegawa M, Fujimoto Y, Lucas PC, Nakano H, Fukase K, Núñez G and Inohara N: A critical role of RICK/RIP2 polyubiquitination in Nod-induced NF-kappaB activation. EMBO J 27: 373-383, 2008.

16. Nomura F, Kawai T, Nakanishi K and Akira S: NF-kappaB activation through IKK-i-dependent I-TRAF/TANK phosphorylation. Genes Cells 5: 191-202, 2000.

17. Terra X, Palozza P, Fernandez-Larrea J, Ardevol A, Blade C Pujadas G, Salvado J, Arola L and Blay MT: Procyanidin dimer B1 and trimer C1 impair inflammatory response signalling in human monocytes. Free Radic Res 45: 611-619, 2011.

18. Nakanishi A, Wada Y, Kitagishi Y and Matsuda S: Link between PI3K/AKT/PTEN pathway and NOX protein in diseases. Aging Dis 5: 203-211, 2014.

19. Korbecki J, Baranowska-Bosiacka I, Gutowska I and Chlubek D: The effect of reactive oxygen species on the synthesis of prostanoids from arachidonic acid. J Physiol Pharmacol 64: 409-421, 2013

20. Tamrakar AK, Schertzer JD, Chiu TT, Foley KP, Bilan PJ, Philpott DJ and Klip A: NOD2 activation induces muscle cell-autonomous innate immune responses and insulin resistance. Endocrinology 151: 5624-5637, 2010.

21. Hales EC, Taub JW and Matherly LH: New insights into Notch1 regulation of the PI3K-AKT-mTOR1 signaling axis: targeted therapy of $\gamma$-secretase inhibitor resistant T-cell acute lymphoblastic leukemia. Cell Signal 26: 149-161, 2014.

22. Johnson SE, Shah N, Bajer AA and LeBien TW: IL-7 activates the phosphatidylinositol 3-kinase/AKT pathway in normal human thymocytes but not normal human B cell precursors. J Immunol 180: 8109-8117, 2008
23. Okumura N, Yoshida H, Kitagishi Y, Murakami M, Nishimura Y and Matsuda S: PI3K/AKT/PTEN signaling as a molecular target in leukemia angiogenesis. Adv Hematol 2012: 843085, 2012.

24. Theodoropoulou M and Stalla GK: Somatostatin receptors: from signaling to clinical practice. Front Neuroendocrinol 34: 228-252, 2013.

25. Liao XH, Buggey J and Kimmel AR: Chemotactic activation of Dictyostelium AGC-family kinases AKT and PKBR1 requires separate but coordinated functions of PDK1 and TORC2. J Cell Sci 123: 983-992, 2010.

26. Kirkegaard T, Witton CJ, Edwards J, et al: Molecular alterations in AKT1, AKT2 and AKT3 detected in breast and prostatic cancer by FISH. Histopathology 56: 203-211, 2010.

27. Robertson GP: Functional and therapeutic significance of Akt deregulation in malignant melanoma. Cancer Metastasis Rev 24: 273-285, 2005.

28. Hodgkinson CP, Sale EM and Sale GJ: Characterization of PDK2 activity against protein kinase B gamma. Biochemistry 41: 10351-10359, 2002

29. Bartolomé A, Guillén C and Benito M: Role of the TSC1-TSC2 complex in the integration of insulin and glucose signaling involved in pancreatic beta-cell proliferation. Endocrinology 151: 3084-3094, 2010.

30. Jastrzebski K, Hannan KM, Tchoubrieva EB, Hannan RD and Pearson RB: Coordinate regulation of ribosome biogenesis and function by the ribosomal protein S6 kinase, a key mediator of mTOR function. Growth Factors 25: 209-226, 2007.

31. Brand C, Cipok M, Attali V, Bak A and Sampson SR: Protein kinase Cdelta participates in insulin-induced activation of PKB via PDK1. Biochem Biophys Res Commun 349: 954-962, 2006.

32. Kim YM, Seo YH, Park CB, Yoon SH and Yoon G: Roles of GSK3 in metabolic shift toward abnormal anabolism in cell senescence. Ann N Y Acad Sci 1201: 65-71, 2010.

33. Downes CP, Perera N, Ross S and Leslie NR: Substrate specificity and acute regulation of the tumour suppressor phosphatase, PTEN. Biochem Soc Symp: 69-80, 2007.

34. Kong D and Yamori T: Advances in development of phosphatidylinositol 3-kinase inhibitors. Curr Med Chem 16: 2839-2854, 2009.

35. Chen Y, Wang SM, Wu JC and Huang SH: Effects of PPARgamma agonists on cell survival and focal adhesions in a Chinese thyroid carcinoma cell line. J Cell Biochem 98: 1021-1035, 2006.

36. Leslie NR, Batty IH, Maccario H, Davidson L and Downes CP. Understanding PTEN regulation: PIP2, polarity and protein stability. Oncogene 27: 5464-5476, 2008.

37. Sheppard K, Kinross KM, Solomon B, Pearson RB and Phillips WA: Targeting PI3 kinase/AKT/mTOR signaling in cancer. Crit Rev Oncog 17: 69-95, 2012.

38. Choi BH, Kim CG, Lim Y, Shin SY and Lee YH: Curcumin down-regulates the multidrug-resistance mdrlb gene by inhibiting the PI3K/Akt/NF kappa B pathway. Cancer Lett 259: 111-118, 2008.

39. Li L, Wei XH, Pan YP, Li HC, Yang H, He QH, Pang Y, Shan Y, Xiong FX, Shao GZ and Zhou RL: LAPTM4B: a novel cancer-associated gene motivates multidrug resistance through efflux and activating PI3K/AKT signaling. Oncogene 29: 5785-5795, 2010.

40. Wu XF, Xu R, Ouyang ZJ, Qian C, Shen Y, Wu XD, Gu YH, $\mathrm{Xu} \mathrm{Q}$ and Sun Y: Beauvericin ameliorates experimental colitis by inhibiting activated $\mathrm{T}$ cells via downregulation of the PI3K/Akt signaling pathway. PLoS One 8: e83013, 2013.

41. Husain N, Tokoro K, Popov JM, Naides SJ, Kwasny MJ and Buchman AL: Neopterin concentration as an index of disease activity in Crohn's disease and ulcerative colitis. J Clin Gastroenterol 47: 246-251,2013.

42. Das A, Xi L and Kukreja RC: Protein kinase G-dependent cardioprotective mechanism of phosphodiesterase-5 inhibition involves phosphorylation of ERK and GSK3beta. J Biol Chem 283 29572-29585, 2008.

43. Molz S, Dal-Cim T, Budni J, Martín-de-Saavedra MD, Egea J, Romero A, del Barrio L, Rodrigues AL, López MG and Tasca CI: Neuroprotective effect of guanosine against glutamate-induced cell death in rat hippocampal slices is mediated by the phosphatidylinositol-3 kinase/Akt/glycogen synthase kinase $3 \beta$ pathway activation and inducible nitric oxide synthase inhibition. J Neurosci Res 89: 1400-1408, 2011.

44. Imai $Y$, Yamagishi H, Ono $Y$ and Ueda $Y$ : Versatile inhibitory effects of the flavonoid-derived PI3K/Akt inhibitor, LY294002, on ATP-binding cassette transporters that characterize stem cells. Clin Transl Med 1: 24, 2012. 
45. Chanoit G, Lee S, Xi J, Zhu M, McIntosh RA, Mueller RA, Norfleet EA and Xu Z: Exogenous zinc protects cardiac cells from reperfusion injury by targeting mitochondrial permeability transition pore through inactivation of glycogen synthase kinase-3beta. Am J Physiol Heart Circ Physiol 295: H1227-H1233, 2008.

46. Jacot JL and Sherris D: Potential therapeutic roles for inhibition of the PI3K/AKT/mTOR pathway in the pathophysiology of diabetic retinopathy. J Ophthalmol 2011: 589813, 2011.

47. Gross ER, Peart JN, Hsu AK, Auchampach JA and Gross GJ: Extending the cardioprotective window using a novel delta-opioid agonist fentanyl isothiocyanate via the PI3-kinase pathway. Am J Physiol Heart Circ Physiol 288: H2744-H2749, 2005.

48. Burke JP, Cunningham MF, Sweeney C, Docherty NG and O'Connell PR: N-cadherin is overexpressed in Crohn's stricture fibroblasts and promotes intestinal fibroblast migration. Inflamm Bowel Dis 17: 1665-1673, 2011.

49. Kuehn HS, Swindle EJ, Kim MS, Beaven MA, Metcalfe DD and Gilfillan AM: The phosphoinositide 3-kinase-dependent activation of Btk is required for optimal eicosanoid production and generation of reactive oxygen species in antigen-stimulated mast cells. J Immunol 181: 7706-7712, 2008.

50. Lee SB, Cho ES, Yang HS, Kim H and Um HD: Serum withdrawal kills U937 cells by inducing a positive mutual interaction between reactive oxygen species and phosphoinositide 3-kinase. Cell Signal 17: 197-204, 2005.

51. Huang S: A new clue to explain resistance to mTOR inhibitors. Cell Cycle 11: 844, 2012.

52. Alvarado Y, Mita MM, Vemulapalli S, Mahalingam D and Mita AC: Clinical activity of mammalian target of rapamycin inhibitors in solid tumors. Target Oncol 6: 69-94, 2011.

53. Dibble CC and Manning BD: Signal integration by mTORC1 coordinates nutrient input with biosynthetic output. Nat Cell Biol 15: 555-564, 2013

54. Guo Y and Kwiatkowski DJ: Equivalent benefit of rapamycin and a potent mTOR ATP-competitive inhibitor, MLN0128 (INK128), in a mouse model of tuberous sclerosis. Mol Cancer Res 11: 467-473, 2013

55. Massey DC, Bredin F and Parkes M: Use of sirolimus (rapamycin) to treat refractory Crohn's disease. Gut 57: 1294-1296, 2008.

56. Yin H, Li X, Zhang B, Liu T, Yuan B, Ni Q, Hu S and Gu H: Sirolimus ameliorates inflammatory responses by switching the regulatory $\mathrm{T} / \mathrm{T}$ helper type 17 profile in murine colitis. Immunology 139: 494-502, 2013.

57. Albiges L, Chamming's F, Duclos B, Stern M, Motzer RJ, Ravaud A and Camus P: Incidence and management of mTOR inhibitor-associated pneumonitis in patients with metastatic renal cell carcinoma. Ann Oncol 23: 1943-1953, 2012.

58. Waters SM, Coyne GS, Kenny DA and Morris DG: Effect of dietary $n-3$ polyunsaturated fatty acids on transcription factor regulation in the bovine endometrium. Mol Biol Rep 41: 2745-2755, 2014

59. Ghosh S, DeCoffe D, Brown K, Rajendiran E, Estaki M, Dai C, Yip A and Gibson DL: Fish oil attenuates omega-6 polyunsaturated fatty acid-induced dysbiosis and infectious colitis but impairs LPS dephosphorylation activity causing sepsis. PLoS One 8: e55468, 2013.

60. Tyagi A, Kumar U, Reddy S, Santosh VS, Mohammed SB, Ehtesham NZ and Ibrahim A: Attenuation of colonic inflammation by partial replacement of dietary linoleic acid with $\alpha$-linolenic acid in a rat model of inflammatory bowel disease. Br J Nutr 108: 1612-1622, 2012.

61. Zhang Z, Zhang C, Wang H, Zhao J, Liu L, Lee J, He Y and Zheng Q: $n-3$ polyunsaturated fatty acids prevents atrial fibrillation by inhibiting inflammation in a canine sterile pericarditis model. Int J Cardiol 153: 14-20, 2011.
62. Nauroth JM, Liu YC, Van Elswyk M, Bell R, Hall EB, Chung G and Arterburn LM: Docosahexaenoic acid (DHA) and docosapentaenoic acid (DPAn-6) algal oils reduce inflammatory mediators in human peripheral mononuclear cells in vitro and paw edema in vivo. Lipids 45: 375-384, 2010.

63. Bassaganya-Riera J, Hontecillas R, Horne WT, Sandridge M, Herfarth $\mathrm{HH}$, Bloomfeld R and Isaacs KL: Conjugated linoleic acid modulates immune responses in patients with mild to moderately active Crohn's disease. Clin Nutr 31: 721-727, 2012.

64. Gravaghi C, La Perle KM, Ogrodwski P, Kang JX, Quimby F, Lipkin $M$ and Lamprecht SA: Cox-2 expression, PGE(2) and cytokines production are inhibited by endogenously synthesized n-3 PUFAs in inflamed colon of fat-1 mice. J Nutr Biochem 22: 360-365, 2011

65. Liu HQ, Qiu Y, Mu Y, Zhang XJ, Liu L, Hou XH, Zhang L, Xu XN, Ji AL, Cao R, Yang RH and Wang F: A high ratio of dietary n-3/n- 6 polyunsaturated fatty acids improves obesity-linked inflammation and insulin resistance through suppressing activation of TLR4 in SD rats. Nutr Res 33: 849-858, 2013.

66. Lee JY, Ye J, Gao Z, Youn HS, Lee WH, Zhao L, Sizemore N and Hwang DH: Reciprocal modulation of Toll-like receptor-4 signaling pathways involving MyD88 and phosphatidylinositol 3-kinase/AKT by saturated and polyunsaturated fatty acids. J Biol Chem 278: 37041-37051, 2003.

67. Aggarwal BB, Gupta SC and Sung B: Curcumin: an orally bioavailable blocker of TNF and other pro-inflammatory biomarkers. Br J Pharmacol 169: 1672-1692, 2013.

68. Song $\mathrm{Y}, \mathrm{Ge} \mathrm{W}$, Cai $\mathrm{H}$ and Zhang $\mathrm{H}$ : Curcumin protects mice from coxsackievirus B3-induced myocarditis by inhibiting the phosphatidylinositol $3 \mathrm{kinase} / \mathrm{Akt} /$ nuclear factor- $\mathrm{\kappa B}$ pathway. J Cardiovasc Pharmacol Ther 18: 560-569, 2013.

69. Fontani F, Marcucci T, Picariello L, Tonelli F, Vincenzini MT and Iantomasi T: Redox regulation of MMP-3/TIMP-1 ratio in intestinal myofibroblasts: effect of $\mathrm{N}$-acetylcysteine and curcumin. Exp Cell Res 323: 77-86, 2014.

70. Krebs S, Omer TN and Omer B: Wormwood (Artemisia absinthium) suppresses tumour necrosis factor alpha and accelerates healing in patients with Crohn's disease-a controlled clinical trial. Phytomedicine 17: 305-309, 2010.

71. Omer B, Krebs S, Omer H and Noor TO: Steroid-sparing effect of wormwood (Artemisia absinthium) in Crohn's disease: a double-blind placebo-controlled study. Phytomedicine 14: 87-95, 2007.

72. Son JE, Lee E, Seo SG, Lee J, Kim JE, Kim J, Lee KW and Lee HJ: Eupatilin, a major flavonoid of Artemisia, attenuates aortic smooth muscle cell proliferation and migration by inhibiting PI3K, MKK3/6, and MKK4 activities. Planta Med 79: 1009-1016, 2013.

73. Yo YT, Shieh GS, Hsu KF, Wu CL and Shiau AL: Licorice and licochalcone-A induce autophagy in LNCaP prostate cancer cells by suppression of Bcl-2 expression and the mTOR pathway. J Agric Food Chem 57: 8266-8273, 2009.

74. Yang JY, Della-Fera MA, Rayalam S and Baile CA: Enhanced effects of xanthohumol plus honokiol on apoptosis in 3T3-L1 adipocytes. Obesity (Silver Spring) 16: 1232-1238, 2008.

75. Liu H, Zang C, Emde A, et al: Anti-tumor effect of honokiol alone and in combination with other anti-cancer agents in breast cancer. Eur J Pharmacol 591: 43-51, 2008.

76. Roy S, Yu Y, Padhye SB, Sarkar FH and Majumdar AP: Difluorinated-curcumin (CDF) restores PTEN expression in colon cancer cells by down-regulating miR-21. PLoS One 8: e68543, 2013

77. Yoshida H, Okumura N, Kitagishi Y, Nishimura Y and Matsuda S: Ethanol extract of rosemary repressed PTEN expression in K562 culture cells. Int J Appl Biol Pharm Technol 2: 316-322, 2011. 